\begin{tabular}{|c|l|}
\hline Title & Uniform local solvability for the Navier-Stokes equations with the Coriolis force \\
\hline Author(s) & Giga, Y oshikazu; Inui, Katsuya; Mahal ov, A lex; Matsui, Shin'ya \\
\hline Citation & Hokkaido University Preprint Series in Mathematics, 736, 1-15 \\
\hline Issue Date & 2005 \\
\hline DOI & 10.14943/83886 \\
\hline Doc URL & http://hdl.handle.net/2115/69544 \\
\hline Type & bulletin (article) \\
\hline File Information & pre736.pdf \\
\hline
\end{tabular}

Instructions for use 


\title{
Uniform local solvability for the Navier-Stokes equations with the Coriolis force
}

Dedicated to Professor Joel A. Smoller on the occasion of his 65 th birthday.

Yoshikazu Giga ${ }^{1}$, Katsuya Inui ${ }^{2}$, Alex Mahalov ${ }^{3}$ and Shin'ya Matsui ${ }^{4}$

${ }^{1}$ Graduate School of Mathematics Sciences, University of Tokyo,

3-8-1 Komaba, Meguro-ku, Tokyo, 153-8914 Japan, Facsimile (81)-3-5465-7023,

labgiga@ms.u-tokyo.ac.jp

2 Department of Mathematics, Keio University,

3-14-1 Hiyoshi, Kohoku-ku, Yokohama, Kanagawa, 223-8522 Japan, Facsimile (81)-45-566-1642, inui@math.keio.ac.jp

${ }^{3}$ Department of Mathematics, Arizona State University, Tempe, AZ 85287-1804, USA, Facsimile (1)-480-357-9791, mahalov@asu.edu

${ }^{4}$ Department of Media Information Science, Hokkaido Information University, Ebetsu 069-8585, Japan, Facsimile (81)-11-384-0134, matsui@do-johodai.ac.jp

\begin{abstract}
The unique local existence is established for the Cauchy problem of the incompressible Navier-Stokes equations with the Coriolis force. The Coriolis operator restricted to divergence free vector fields is a zero order pseudodifferential operator with the skew-symmetric matrix symbol related to the Riesz operator. It leads to the additional term in the Navier-Stokes equations which has real parameter being proportional to the speed of rotation. For initial data as Fourier preimage of the space of all finite Radon measures with no point mass at the origin we prove uniform estimate for the existence time in the speed of rotation.
\end{abstract}

\section{Introduction}

In this paper we consider the Cauchy problem for the three dimensional Navier-Stokes equations with the Coriolis force:

$$
u_{t}+(u \cdot \nabla) u+\Omega e_{3} \times u-\Delta u=-\nabla p, \quad \nabla \cdot u=0,\left.\quad u\right|_{t=0}=u_{0},
$$

where $u=u(x, t)=\left(u_{1}(x, t), u_{2}(x, t), u_{3}(x, t)\right)$ is the unknown velocity vector field and $p=p(x, t)$ is the unknown scalar pressure at the point $x=$ 
$\left(x_{1}, x_{2}, x_{3}\right) \in \mathbb{R}^{3}$ in space and time $t>0$, while $u_{0}=u_{0}(x)$ is the given initial velocity vector field. Here, the real constant $\Omega$ represents the speed of rotation around the vertical unit vector $e_{3}=(0,0,1)$ and it is called the Coriolis parameter. By $\times$ we denote the exterior product, hence, the Coriolis term is represented by $e_{3} \times u \equiv \mathbf{J} u$ with the corresponding skew-symmetric $3 \times 3$ matrix $\mathbf{J}$.

For the equations (1.1) global existence and regularity results for large fixed $\Omega$ were obtained in $L^{2}$ setting for the periodic domains such as cylinder with infinite length rotating axis or spatial lattices ([2], [6]). In this regard, for the Euler equations, Nicolaenko, Bardos, Golse and the third author [7] proved uniform local existence and long-time regularity for initial data in $H^{4}$.

In the case of unbounded domains without periodicity nor decay at space infinity Sawada [9] and Hieber and Sawada [5] proved unique local existence for initial data in a homogeneous Besov space $\dot{B}_{\infty, 1}^{0}$ which is strictly smaller than $B U C$, the space of all bounded uniformly continuous functions (see also [3] for recent improvement). However, they do not get uniform estimate for the existence time in the speed of rotation $\Omega \in \mathbb{R}$, the precondition to consider fast singular oscillating limits $\Omega \rightarrow+\infty$ and proving global existence for large fixed $\Omega$.

In this paper we take initial data in a new space $F M_{0}$ (Fourier preimage of the space of all finite Radon measures with no point mass at the origin) that ensures uniform local solvability of (1.1). The space for initial data $F M_{0}$ is strictly smaller than the homogeneous Besov space $\dot{B}_{\infty, 1}^{0}$ and its precise definition will be given in section 2 .

Our key observation is a bound of $\exp \left(t R_{j}\right)$ in the space $F M_{0}$ uniform in time $t$, where $R_{j}$ is a Riesz operator. This enables us to construct a local solution uniform in $\Omega$.

Now we state the main result of this paper.

Theorem 1.1. Assume that $u_{0} \in F M_{0}$ with div $u_{0}=0$. Then there exist $T_{0}\left(\geq c /\left\|u_{0}\right\|_{F M}^{2}\right)>0$ independent of the Coriolis parameter $\Omega$ and a unique mild solution $u=u(t) \in C\left(\left[0, T_{0}\right] ; F M_{0}\right)$ of $(1.1)$, where $c>0$ is a numerical constant (given in Remark 3.1). Moreover, the solution $u(t)$ belongs to $C\left(\left[0, T_{0}\right] ; F M\right)$.

From the above theorem on a mild solution we also have the following theorem on existence and uniqueness of the classical solution of (1.1). The proof is carried out in the same manner as [3].

Theorem 1.2. Assume that $u_{0} \in F M_{0}$ with div $u_{0}=0$.

(1) Let $u=u(t)$ be the mild solution obtained in Theorem 1.1. If we set the pressure $p=p(x, t)$ as

$$
\partial_{i} p(t)=\partial_{i} \sum_{j, k=1}^{3} R_{j} R_{k} u^{j} u^{k}(t)+\Omega R_{i}\left(R_{2} u^{1}-R_{1} u^{2}\right)(t)
$$


for $t>0$ and $i=1,2,3$, then the pair $(u, \nabla p)$ is a classical solution of (1.1).

(2) Let $u \in L^{\infty}\left((0, T) \times \mathbb{R}^{3}\right)$ and $p \in L_{\text {loc }}^{1}([0, T) ; B M O)$ be a solution of (1.1) in a distributional sense for some $T>0$. Then the pair $(u, \nabla p)$ is unique. Moreover, the relation (1.2) holds.

Before concluding the introduction we remark that $u(x, t)$ is almost periodic in $x$ if $u_{0}$ is almost periodic. This can be proved along the line of [4, Section 3] if one observes that $F M$-norm is invariant under translation in spatial variables $x \in \mathbb{R}^{3}$.

\section{Key function spaces}

In this section we introduce a function space on which the semigroup generated by the Riesz operator is uniformly bounded; moreover, it contains various almost periodic functions, not necessarily periodic. Furthermore, operation of spatial averaging is defined for the elements of this space. The latter property and uniform boundedness with respect to spatial translations and rotations are essential in studies of statistical properties of turbulence (e.g. [8], [11]). We now recall an important connection between the Coriolis rotation operator restricted to divergence free vector fields and classical Riesz operators. For the sake of clarity of notations, we present formulas for symbols of the corresponding pseudo-differential operators in $\mathbb{R}^{3}$ which is the main case. Let $\mathbf{P}$ be the projection operator on divergence free vector fields, represented by $\mathbf{P}=\left(P_{i j}\right)_{i j}=\left(\delta_{i j}+R_{i} R_{j}\right)_{1 \leq i, j \leq 3}$. Here, $\delta_{i j}$ denotes the Kronecker delta. Applying $\mathbf{P}$ to (1.1), we note that the Coriolis term is represented by the Coriolis operator $\mathbf{S}=\mathbf{P J P}(\mathbf{P J P} u=\mathbf{P J} u$ since $u$ is solenoidal).

Let $\xi=\left(\xi_{1}, \xi_{2}, \xi_{3}\right) \in \mathbb{R}^{3}$. The operator $\mathbf{S}$ is a zero order pseudo-differential operator with the $3 \times 3$ matrix symbol $\mathbf{S}(\xi)([1]$, [3]):

$$
\sigma(\mathbf{S}) \equiv \mathbf{S}(\xi)=\frac{\xi_{3}}{|\xi|} \mathbf{R}(\xi)
$$

where $\mathbf{R}(\xi)$ is the skew-symmetric matrix such that $\mathbf{R}(\xi) v=\frac{1}{\mid \xi} \xi \times v$ for every vector $v \in \mathbb{R}^{3}$. The symbol of the operator $\exp (\Omega \mathbf{S} t)$ is given by

$$
\exp (\Omega \mathbf{S}(\xi) t)=\cos \left(\frac{\xi_{3}}{|\xi|} \Omega t\right) \mathbf{I}+\sin \left(\frac{\xi_{3}}{|\xi|} \Omega t\right) \mathbf{R}(\xi)
$$

where $\mathbf{I}$ is the identity matrix. We remark that dependence on the parameter $\Omega$ appears only in scalar terms $\cos \left(\frac{\xi_{3}}{|\xi|} \Omega t\right)$ and $\sin \left(\frac{\xi_{3}}{|\xi|} \Omega t\right)$, which are functions of the classical scalar Riesz operator $R_{3}, \sigma\left(R_{3}\right)=\frac{i \xi_{3}}{|\xi|}$. Then our goal in this section is to construct function spaces for initial data in which Fourier operators with the symbols $e^{ \pm i \frac{\xi_{3}}{|\xi|} \Omega t}$ are uniformly bounded in $\Omega$.

\subsection{Spaces of measures}


Let $M=M\left(\mathbb{R}^{n}\right)$ be the space of all complex-valued finite Radon measures on $\mathbb{R}^{n}$. This space is the (complex) Banach space equipped with the total variation norm $\|\cdot\|_{M}$. By the Riesz representation theorem $M$ is identified with the dual space of $C_{\infty}\left(\mathbb{R}^{n}\right)$, the space of all (complex-valued) continuous functions that converge to zero at the space infinity (equipped with the supremum norm $\|\cdot\|_{\infty}$.) Moreover, for $\mu \in M$

$$
\|\mu\|_{M}=\sup \left\{|\langle\mu, \psi\rangle| ; \psi \in C_{\infty}\left(\mathbb{R}^{n}\right),\|\psi\|_{\infty} \leq 1\right\},
$$

where $\langle$,$\rangle denotes a canonical pairing. Since the (Schwartz) space \mathcal{S}=\mathcal{S}\left(\mathbb{R}^{n}\right)$ of all rapidly decreasing functions in $\mathbb{R}^{n}$ is dense in $C_{\infty}\left(\mathbb{R}^{n}\right), \mu \in M$ is regarded as a tempered distribution, i.e. $\mu \in \mathcal{S}^{\prime}=\mathcal{S}^{\prime}\left(\mathbb{R}^{n}\right)$. In other words, $M \subset \mathcal{S}^{\prime}$. Let $|\mu|$ denote the total variation measure of $\mu$. It is a nonnegative Radon measure defined by

$$
|\mu|(O)=\sup \left\{|\langle\mu, \psi\rangle| ; \psi \in C_{0}(O),\|\psi\|_{\infty} \leq 1\right\}
$$

for an open set $O$, where $C_{0}(O)$ denotes the space of all (complex-valued) continuous functions with compact support in $O$. Since $C_{0}\left(\mathbb{R}^{n}\right)$ is dense in $C_{\infty}\left(\mathbb{R}^{n}\right)$, we see that $|\mu|\left(\mathbb{R}^{n}\right)=\|\mu\|_{M}$. For $\mathbb{C}^{d}$-valued (complex vector-valued) finite Radon measure $\mu=\left(\mu_{1}, \cdots, \mu_{d}\right)$ we still denote its total variation measure $|\mu|$ by the above duality by understanding that $\|\psi\|_{\infty}=\||\psi|\|_{\infty}$ for $\psi=\left(\psi_{1}, \cdots, \psi_{d}\right)$, where $|\cdot|$ denotes the Euclidean norm in $\mathbb{R}^{d}$. The totality of $\mathbb{C}^{d}$-valued finite Radon measures on $\mathbb{R}^{n}$ is denoted by $M^{d}=\left(M\left(\mathbb{R}^{n}\right)\right)^{d}$. (We often write $M^{d}$ simply by $M$ unless confusion is caused.) There are several ways to define a norm in $\left(M\left(\mathbb{R}^{n}\right)\right)^{d}$. The most convenient one for our purpose is

$$
\|\mu\|_{M^{d}}=|\mu|\left(\mathbb{R}^{n}\right) .
$$

Another norm we use here is

$$
\|\mu\|_{M^{d}}^{\prime}=\left(\sum_{i=1}^{d}\left\|\mu_{i}\right\|_{M}^{2}\right)^{1 / 2} .
$$

In the case that $\mu_{i}$ is absolutely continuous with respect to the Lebesgue measure, i.e. $\mu_{i}=\rho_{i} d \xi$, then

$$
\|\mu\|_{M^{d}}=\int_{\mathbb{R}^{n}}\left(\sum_{i=1}^{d}\left|\rho_{i}\right|^{2}\right) d \xi,\|\mu\|_{M^{d}}^{\prime}=\left(\sum_{i=1}^{d}\left(\int_{\mathbb{R}^{n}}\left|\rho_{i}\right| d \xi\right)^{2}\right)^{1 / 2} .
$$

As we shall see later these norms are equivalent. For the definition of total variations as well as several elementary properties of measures, the reader is referred to, for example, a book [10] of L. Simon.

For a bounded Borel measurable function $\psi$ and $\mu \in M$ we associate a new Radon measure $\mu\lfloor\psi \in M$ defined by

$$
\left(\mu\lfloor\psi)(O)=\int_{O} \psi(\xi) \mu(d \xi)\right.
$$


If $\psi$ is a characteristic function $\chi_{B}$ of a Borel set $B$, we simply write $\mu \chi \chi_{B}$ by $\mu\lfloor B$. Note that $\psi$ is allowed to be any $|\mu|$-measurable functions. We here prove that above two norms of $M^{d}$ are equivalent. More precisely,

$$
\|\mu\|_{M^{d}}^{\prime} \leq\|\mu\|_{M^{d}} \leq \sqrt{d}\|\mu\|_{M^{d}}^{\prime}, \mu \in M^{d} .
$$

Indeed, let $h_{i}$ be the Radon-Nikodym derivative of $\mu_{i}$ with respect to $|\mu|$; this is $|\mu|$-a.e. well-defined since $\mu_{i}$ is absolutely continuous with respect to $|\mu|$. Then

$$
\|\mu\|_{M^{d}}^{\prime}=\left\{\sum_{i=1}^{d}\left(\int_{\mathbb{R}^{n}}\left|h_{i}\right||\mu|(d \xi)\right)^{2}\right\}^{1 / 2} .
$$

Applying Minkowski's inequality, we have

$$
\|\mu\|_{M^{d}}^{\prime} \leq \int_{\mathbb{R}^{n}}\left(\sum_{i=1}^{d}\left|h_{i}\right|^{2}\right)^{1 / 2}|\mu|(d \xi)=|\mu|\left(\mathbb{R}^{n}\right)=\|\mu\|_{M^{d}}
$$

since $\sum_{i=1}^{d}\left|h_{i}\right|^{2}(\xi)=1$ for $|\mu|$-a.e. $\xi$. If is easy to see that $\|\mu\|_{M^{d}} \leq \sum_{i=1}^{d}\left\|\mu_{i}\right\|_{M}$, so we have $\|\mu\|_{M^{d}} \leq \sqrt{d}\|\mu\|_{M^{d}}^{\prime}$ by Schwarz' inequality.

We next consider an important subspace $M_{0}$ of $M\left(\mathbb{R}^{n}\right)$ for our purpose defined by

$$
M_{0}=\{\mu \in M ; \mu\lfloor\{0\}=0\}
$$

(By $M_{0}^{d}$ we mean $\left.\left(M_{0}\left(\mathbb{R}^{n}\right)\right)^{d}\right)$. In other words $\mu \in M$ belongs to $M_{0}$ if and only if there is no point mass at the origin. The condition $\mu\lfloor\{0\}=0$ is equivalent to say that

$$
\lim _{r \downarrow 0}|\mu|\left(\stackrel{\circ}{B}_{r}(0)\right)=0,
$$

where $\stackrel{\circ}{B}_{r}(0)$ is the open ball of radius $r$ centered at the origin. Thus the space $M_{0}$ is a closed subspace of $M_{0}$. Indeed, if $\mu_{m} \rightarrow \mu$ in $M$ and $\mu_{m}$ satisfies (2.3), then

$$
\begin{aligned}
|\mu|\left(\stackrel{\circ}{B}_{r}(0)\right) & \leq\left|\mu_{m}-\mu\right|\left(\stackrel{\circ}{B}_{r}(0)\right)+\left|\mu_{m}\right|\left(\stackrel{\circ}{B}_{r}(0)\right) \\
& \leq\left\|\mu_{m}-\mu\right\|_{M}+\left|\mu_{m}\right|\left(\stackrel{\circ}{B}_{r}(0)\right) .
\end{aligned}
$$

Sending $r$ to 0 , we have

$$
\underset{r \downarrow 0}{\limsup }|\mu|\left(\stackrel{\circ}{B}_{r}(0)\right) \leq\left\|\mu_{m}-\mu\right\|_{M} .
$$

Since $\mu_{m} \rightarrow \mu$ in $M$, this implies that $\mu$ satisfies (2.3), i.e., $\mu \in M_{0}$.

Lemma 2.1. Let $\sigma \in C\left(\mathbb{R}^{n} \backslash\{0\}\right)$ be bounded on $\mathbb{R}^{n} \backslash\{0\}$. Assume that $\mu \in M_{0}$ and that $\left\{\sigma_{m}\right\}_{m=1}^{\infty} \subset \mathcal{S}\left(\mathbb{R}^{n}\right)$ is an approximate sequence of $\sigma$ in the sense that $\sigma_{m} \rightarrow \sigma$ pointwise in $\mathbb{R}^{n} \backslash\{0\}$ and $\left\|\sigma_{m}\right\|_{\infty}$ is uniformly bounded. Then 
$\left\{\mu\left\lfloor\sigma_{m}\right\}_{m=1}^{\infty} \subset M\right.$ is a convergent sequence in $M_{0}$. Moreover, its limit equals $\left(\mu\left\lfloor\left(\mathbb{R}^{n} \backslash\{0\}\right)\right)\left\lfloor\sigma \in M_{0}\right.\right.$ and is independent of the choice of the approximate sequence $\left\{\sigma_{m}\right\}_{m=1}^{\infty}$.

Remark 2.1. Let $\bar{\sigma}$ be any extension of $\sigma \in C\left(\mathbb{R}^{n} \backslash\{0\}\right)$ to the origin. Since $\mu \in M_{0}$ so that $\mu=\mu\left\lfloor\left(\mathbb{R}^{n} \backslash\{0\}\right)\right.$, we see that $\left(\mu\left\lfloor\left(\mathbb{R}^{n} \backslash\{0\}\right)\right)\lfloor\sigma=\mu\lfloor\bar{\sigma}\right.$. In particular, the measure $\mu\lfloor\bar{\sigma}$ is independent of the way of the extension of $\sigma$.

We shall denote $\left(\mu\left\lfloor\left(\mathbb{R}^{n} \backslash\{0\}\right)\right)\left\lfloor\sigma\right.\right.$ simply by $\mu\left\lfloor\sigma \in M_{0}\right.$ for $\mu \in M_{0}$. (The measure $\mu\lfloor\bar{\sigma}$ in general depends on the value $\bar{\sigma}\lfloor 0$ when $\mu \in M$.)

Proof. By the Lebesgue dominated convergence theorem we see that

$$
\lim _{m \rightarrow \infty} \int_{\mathbb{R}^{n} \backslash\{0\}}\left|\sigma_{m}-\sigma\right| \mu(d \xi)=0 .
$$

Since $\mu \in M_{0}$ so that $\mu\left\lfloor\sigma_{m}=\left(\mu\left\lfloor\sigma_{m}\right)\left\lfloor\left(\mathbb{R}^{n} \backslash\{0\}\right)\right.\right.\right.$, this implies

$$
\mu\left\lfloor\sigma _ { m } \rightarrow \left(\mu \lfloor ( \mathbb { R } ^ { n } \backslash \{ 0 \} ) ) \left\lfloor\sigma \in M_{0} .\right.\right.\right.
$$

\subsection{Fourier images and Riesz operator}

We shall consider Fourier (pre)image of spaces $M$ and $M_{0}$ introduced above. For $f \in \mathcal{S}$ we define its Fourier transform and its inverse Fourier transform by

$$
\begin{aligned}
& F f(\xi)=\hat{f}(\xi)=\frac{1}{(2 \pi)^{n / 2}} \int_{\mathbb{R}^{n}} e^{-i \xi \cdot x} f(x) d x, \\
& F^{-1} f(x)=\check{f}(x)=\frac{1}{(2 \pi)^{n / 2}} \int_{\mathbb{R}^{n}} e^{i x \cdot \xi} f(\xi) d \xi,
\end{aligned}
$$

where $\xi \cdot x$ denotes the standard inner product in $\mathbb{R}^{n}$ and $i=\sqrt{-1}$. The operator $F$ and $F^{-1}$ can be extended to an isomorphism of $\mathcal{S}^{\prime}$ as topological vector spaces. Let $F M$ be the image of $M$ by $F$. It is the preimage of $M$ by $F^{-1}$. Since $F^{-1} f(x)=(F f)(-x)$, the space $F M$ is also the preimage of $M$ by $F$, i.e.,

$$
F M=\left\{f \in \mathcal{S}^{\prime} ; \hat{f} \in M\right\} .
$$

This space is a Banach space equipped with the norm

$$
\|f\|_{F M}=(2 \pi)^{-n / 2}\|\hat{f}\|_{M} .
$$

Let $F M_{0}$ be the closed subspace of $F M$ of the form

$$
F M_{0}=\left\{f \in \mathcal{S}^{\prime} ; \hat{f} \in M_{0}\right\}
$$

The space $F M$ is included in the space $B U C$, the space of all bounded uniformly continuous functions. The space $F M_{0}$ does not contain nonzero constant function. In fact, $F M$ has a topological direct sum decomposition of the form

$$
F M=F M_{0} \oplus \mathbb{C}
$$


where $\mathbb{C}$ denotes the space of all (complex) constant functions. Moreover, as we shall see in Appendix, $F M_{0}$ is strictly included in the Besov space $\dot{B}_{\infty, 1}^{0}$. However, $F M_{0}$ still includes various almost periodic functions not necessarily periodic. For example,

$$
f(x)=\sum_{j=1}^{\infty} \alpha_{j} e^{i \lambda_{j} \cdot x} \quad \lambda_{j} \in \mathbb{R}^{n} \backslash\{0\}, \quad \alpha_{j} \in \mathbb{C}, \quad \lambda_{j} \neq \lambda_{k} \text { if } j \neq k
$$

belongs to $F M_{0}$ if $\sum_{j=1}^{\infty}\left|\alpha_{j}\right|<\infty$. Indeed,

$$
\hat{f}(\xi)=(2 \pi)^{n / 2} \sum_{j=1}^{\infty} \alpha_{j} \delta\left(\xi-\lambda_{j}\right) \in M_{0} .
$$

Its norm $\|f\|_{F M}=\sum_{j=1}^{\infty}\left|\alpha_{j}\right|$ if $\lambda_{j} \neq \lambda_{k}$ for $j \neq k$.

We next study an operator whose symbol may not be continuous at the origin. We shall prove that

$$
(\Sigma f)(x)=F^{-1}(\sigma(\xi)((F f)(\xi)))(x)
$$

is a well-defined operator for $f \in F M_{0}$ if the symbol $\sigma \in C\left(\mathbb{R}^{n} \backslash\{0\}\right)$ is bounded. We approximate $\sigma$ by $\left\{\sigma_{m}\right\} \subset \mathcal{S}$ such that $\left\|\sigma_{m}\right\|_{\infty}$ is bounded and $\sigma_{m} \rightarrow \sigma$ pointwise in $\mathbb{R}^{n} \backslash\{0\}$. (This is of course possible.) The quantity $F^{-1}\left(\sigma_{m} F f\right)$ is well-defined quantity in $\mathcal{S}^{\prime}$ if $f \in \mathcal{S}^{\prime}$. By Lemma $2.1 F^{-1} \sigma_{m} F f$ converges to $F^{-1}\left((F f)\lfloor\sigma)\right.$ in $F M_{0}$ if $f \in F M_{0}$. So we shall define $\Sigma f$ by

$$
\Sigma f=F^{-1}((F f)\lfloor\sigma) .
$$

Lemma 2.2. Assume that $\sigma \in C\left(\mathbb{R}^{n} \backslash\{0\}\right)$ is bounded in $\mathbb{R}^{n} \backslash\{0\}$. Then $\Sigma$ in (2.3) is a bounded linear operator in $F M_{0}$ and

$$
\|\Sigma f\|_{F M} \leq\|\sigma\|_{\infty}\|f\|_{F M}
$$

for $f \in F M_{0}$. If, moreover $\sigma$ is continuous at the origin, then $\Sigma$ is a bounded linear operator in $F M$ and (2.4) holds for all $f \in F M$.

This is clear by definition of the norm. As a simple application we obtain the boundedness on $F M_{0}$ of the Riesz operator

$$
\left(R_{j} f\right)=F^{-1}\left(F f\left\lfloor\sigma\left(R_{j}\right)\right), \sigma\left(R_{j}\right)=i \xi_{j} /|\xi|, j=1, \cdots, n\right.
$$

although the symbol $\sigma\left(R_{j}\right)$ is not continuous at $\xi=0$.

Lemma 2.3. The Riesz operator $R_{j}$ is bounded in $F M_{0}$ for $j=1,2, \cdots, n$. Moreover,
(i) $\left\|R_{j} f\right\|_{F M} \leq\|f\|_{F M} \quad t>0$,

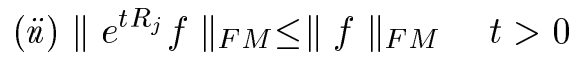


for all $f \in F M_{0}$.

\subsection{Multiplication and heat operators}

It is rather clear that $F M$ is an algebra whose unit is a constant function 1. The space $F M_{0}$ is not an algebra since the multiplication of $e^{i \lambda \cdot x}$ and $e^{-i \lambda \cdot x}$ equals $1 \notin F M_{0}$ for $\lambda \in \mathbb{R}^{n} \backslash\{0\}$.

Lemma 2.4. If $f$ and $g$ are in $F M$, so is $f g$. The norm of the unit 1 equals 1 . Moreover,

$$
\|f g\|_{F M} \leq\|f\|_{F M}\|g\|_{F M} .
$$

Proof. Since $\widehat{f g}=(2 \pi)^{-n / 2} \hat{f} * \hat{g}$, Fubini's theorem implies that

$$
\|\widehat{f g}\|_{M} \leq(2 \pi)^{-n / 2}\|\hat{f}\|_{M}\|\hat{g}\|_{M}
$$

which yields the desired estimate. Since $\hat{1}=(2 \pi)^{n / 2} \delta(x)$, by definition $\|1\|_{F M}=1$.

We next study the heat semigroup on $F M$ and $F M_{0}$. For $f \in F M$ we define

$$
e^{t \Delta} f=F^{-1}\left(e^{-t|\xi|^{2}}(F f)\right)=F^{-1}\left(\hat{f}\left\lfloor e^{-t|\xi|^{2}}\right), \quad t \geq 0 .\right.
$$

Similarly, we also define

$$
\partial_{x_{j}} e^{t \Delta} f=F^{-1}\left(i \xi_{j} e^{-t|\xi|^{2}}(F f)\right)=F^{-1}\left(\hat{f}\left\lfloor i \xi_{j} e^{-t|\xi|^{2}}\right), \quad t \geq 0,\right.
$$

which is of course consistent with $\partial_{x_{j}}\left(e^{t \Delta} f\right)$.

\section{Lemma 2.5.}

(i) The family $\left\{e^{t \Delta}\right\}_{t \geq 0}$ is a bounded $C_{0}$-semigroup in $F M$ and $F M_{0}$. Moreover, $\left\|e^{t \Delta} f\right\|_{F M} \leq\|f\|_{F M}$ for $f \in F M, t \geq 0$.

(ii) Let $A$ be a closed linear operator defined by

$$
A f=\Delta f
$$

for $f \in D(A)=\left\{f \in F M ; \frac{\partial^{2}}{\partial x_{i} \partial x_{j}} f \in F M,(1 \leq i, j \leq n)\right\}$, Then $A$ is the infinitesimal generator of the semigroup $\left\{e^{t \Delta}\right\}_{t \geq 0}$ in FM. The restriction $A$ on $F M_{0}$ (denoted by $A_{0}$ ) is the infinitesimal generator of $\left\{e^{t \Delta}\right\}_{t \geq 0}$ in $F M_{0}$.

(iii) The semigroup $\left\{e^{t \Delta}\right\}_{t \geq 0}$ is an analytic semigroup in $F M$ and $F M_{0}$. Moreover,

$$
\left\|\partial_{x_{j}} e^{t \Delta} f\right\|_{F M} \leq(2 t e)^{-1 / 2}\|f\|_{F M}, f \in F M, t \geq 0, i=1, \cdots, n
$$

and $\partial_{x_{j}} e^{t \Delta} f \in F M_{0}$, where $\partial_{x_{j}}=\partial / \partial x_{j}$. 
Proof. All properties are easy to prove if one works on the Fourier images of $f$ and $e^{t \Delta} f$. Necessary estimates are obtained by Lemma 2.2.

\subsection{Averaging}

An element $f \in F M$ always has a vertical averaging in the sense that

$$
\lim _{L \rightarrow \infty} \frac{1}{2 L} \int_{-L}^{L} f\left(x_{1}, \cdots, x_{n-1}, x_{n}\right) d x_{n}=: f_{a}\left(x^{\prime}\right), \quad x^{\prime}=\left(x_{1}, \cdots, x_{n-1}\right)
$$

exists at least for almost every $x^{\prime} \in \mathbb{R}^{n-1}$. We call the function $f_{a}$ the vertical average of $f$. The function $f-f_{a}$ is denoted by $f^{\perp}$. Actually, we have a stronger result. Let $\chi^{L}$ be a function defined by

$$
\chi^{L}\left(x_{n}\right)=\frac{1}{2 L} \chi_{(-L, L)}\left(x_{n}\right)
$$

and $\chi_{(-L, L)}\left(x_{n}\right)$ is the characteristic function of $(-L, L)$, i.e., $\chi_{(-L, L)}\left(x_{n}\right)=1$ for $x_{n} \in(-L, L)$ and otherwise $\chi^{L}(x)=0$.

Lemma 2.6.

(i) Assume that $f \in F M$ fulfills $\hat{f}\left\lfloor\left\{\xi_{n}=0\right\}=0\right.$. (In particular $f \in F M_{0}$ ). Then $\chi^{L} * f \rightarrow 0$ in $F M$ as $L \rightarrow \infty$.

(i) Assume that $f \in F M$ fulfills $\hat{f}\left\lfloor\left\{\xi_{n} \neq 0\right\}=0\right.$. (This means $f$ is independent of $x_{n}$.) Then $\chi^{L} * f=f$ for all $L>0$.

(iii) The space $F M$ has a direct sum decomposition

$$
\begin{array}{ll} 
& F M=F M^{\perp} \oplus F M_{a} \\
\text { with } & F M^{\perp}=\left\{f \in F M ; \hat{f}\left\lfloor\left\{\xi_{n} \neq 0\right\}=0\right\}\right. \\
& F M_{a}=\left\{f \in F M ; \hat{f}\left\lfloor\left\{\xi_{n}=0\right\}=0\right\} .\right.
\end{array}
$$

Proof.

(i) Since $\hat{\chi}^{L}\left(\xi_{n}\right)=\sin \left(L \xi_{n}\right) / L \xi_{n}$, we see that $\hat{\chi}^{L}\left(\xi_{n}\right) \rightarrow 0$ as $L \rightarrow \infty$ for $\xi_{n} \neq 0$ and $\left|\hat{\chi}^{L}\left(\xi_{n}\right)\right| \leq 1$. Since

$$
\left\|f * \chi^{L}\right\|_{F M}=\left\|\hat{\chi}^{L} \hat{f}\right\|_{M}=\|\left(\hat { f } \lfloor \chi ^ { L } ) \left\lfloor\left\{\xi_{n} \neq 0\right\} \|_{M},\right.\right.
$$

the Lebesgue dominated convergence theorem yields that $\chi^{L} * f \rightarrow 0$ for $f \in F M$ if $\hat{f}\left\lfloor\left\{\xi_{n}=0\right\}=0\right.$.

(ii) This is trivial.

(iii) For $f \in F M$ we define mappings

$$
f \mapsto F^{-1}\left(\hat{f}\left\lfloor\left\{\xi_{n}=0\right\}\right), \quad f \mapsto F^{-1}\left(\hat{f}\left\lfloor\left\{\xi_{n} \neq 0\right\}\right)\right.\right.
$$

which give projections to $F M^{\perp}$ and $F M_{a}$. 


\subsection{Divergence free spaces}

We shall study the space of vector fields in $\mathbb{R}^{n}$ whose components belong to $F M$. To simplify notation we still denote such a space by $F M$. We shall apply such convention for other spaces like $F M_{0}, F M^{\perp}, F M_{a}$.

Let $\mathbf{P}$ be defined by

$$
(\mathbf{P} f)_{j}=\sum_{k=1}^{n} F^{-1}\left(\hat{f}_{k}\left\lfloor\sigma_{j k}(\mathbf{P})\right) \quad \text { for } \quad f=\left(f_{1}, \cdots, f_{n}\right) \in F M_{0}, j=1, \cdots, n\right.
$$

with the symbol $\sigma_{j k}(\mathbf{P})(\xi)=\delta_{j k}-\xi_{j} \xi_{k} /|\xi|^{2}, \xi \in \mathbb{R}^{n}$. By Lemma 2.2 this operator is bounded in $F M_{0}$. Let $P F M_{0}$ denote the $\mathbf{P}$-image of $F M_{0}$. We set $\mathbf{Q}=I-\mathbf{P}$ and observe that

$$
(\mathbf{Q} f)_{j}=\sum_{k=1}^{n} F^{-1}\left(\hat{f}_{k}\left\lfloor-\xi_{j} \xi_{k} /|\xi|^{2}\right) \text { for } f=\left(f_{1}, \cdots, f_{n}\right) \in F M_{0} .\right.
$$

Let $Q F M_{0}$ denote the Q-image of $F M_{0}$. As usual, we have a Helmholtz decomposition of $F M_{0}$.

Lemma 2.7. The space $F M_{0}$ has a topological direct sum decomposition

$$
F M_{0}=P F M_{0} \oplus Q F M_{0} .
$$

The space $P F M_{0}$ agrees with the space of all divergence free vector fields in $F M_{0}$.

Proof. Since $\mathbf{P}^{2}=\mathbf{P}$ so that $\mathbf{P Q}=\mathbf{Q P}=0$ and since $\mathbf{P}$ is bounded in $F M_{0}$ the first statement is clear. If $f$ satisfies $\operatorname{div} f=0$ so that $\sum_{k=1}^{n} \hat{f}_{k}\left\lfloor\xi_{k}=0\right.$, then $\mathbf{Q} f=0$ so $f \in P F M_{0}$. If $f \in P F M_{0}$ so that $f=\mathbf{P} f$, then $\operatorname{div} f=\operatorname{div} \mathbf{P} f=0$ by a calculation of symbols. We shall estimate norms of several operators acting in vector valued $F M$ spaces. For $f \in F M=(F M)^{d}$ we define

$$
\|f\|_{F M}=(2 \pi)^{-n / 2}\|\hat{f}\|_{M^{d}},\|f\|_{F M}^{\prime}=(2 \pi)^{-n / 2}\|\hat{f}\|_{M^{d}}^{\prime}
$$

where $\hat{f}=\left(\hat{f}_{1}, \cdots, \hat{f}_{d}\right), f=\left(f_{1}, \cdots, f_{d}\right)$. We shall state a vectorial version of Lemma 2.2. For $d \times d$ complex matrix valued function $\sigma=\left(\sigma_{j k}\right)_{1 \leq j, k \leq d}$ we define

$$
(\Sigma f)_{j}=\sum_{k=1}^{d} F^{-1}\left(\hat{f}_{k}\left\lfloor\sigma_{j k}\right) \quad(j=1, \cdots, d)\right.
$$

instead of (2.3). For $d \times d$ matrix $A$ let $|A|$ be the operator norm form $\mathbb{C}^{d}$ to $\mathbb{C}^{d}$ equipped with standard inner product.

Lemma 2.8. (i) Assume that $\sigma=\left(\sigma_{j k}\right) \in C\left(\mathbb{R}^{n} \backslash\{0\}\right)$ is bounded in $\mathbb{R}^{n} \backslash\{0\}$. Then $\Sigma$ in (2.3) is a bounded linear operator in $F M_{0}$ with an estimate

$$
\|\Sigma f\|_{F M^{d}} \leq\||\sigma|\|_{\infty}\|f\|_{F M^{d}}
$$


for $f \in F M_{0}$. If, moreover $\sigma$ is continuous at the origin, then $\sigma$ is a bounded linear operator in $F M$ satisfying (2.6) for all $f \in F M$.

Proof. It suffices to estimate Fourier transforms. We notice that

$$
\sum_{k=1}^{d} \hat{f}_{k}\left\lfloor\sigma_{j k}=|\hat{f}|\left\lfloor\sum_{k=1}^{d} h_{k} \sigma_{j k},\right.\right.
$$

where $h_{k}$ is the Radon-Nikodym derivative of $\hat{f}_{k}$ with respect to $|\hat{f}|$. Since $|h|(\xi)=1$ for $|\hat{f}|$-a.e. $\xi$, by definition of $\sigma$

$$
\left|\sum_{k=1}^{d} h_{b}(\xi) \sigma_{j k}(\xi)\right| \leq|\sigma(\xi)||h(\xi)|=|\sigma(\xi)|
$$

for $h=\left(h_{1}, \cdots, h_{d}\right)$. We now obtain $(2.6)$ by using the supremum norm of $|\sigma|$.

Lemma 2.8 provides a stronger consequence for $\mathbf{P}$ or $\exp (\mathbf{S} t)$ than that follows from Lemma 2.3.

Lemma 2.9. (i) Let $\mathbf{P}$ be defined in the beginning of $\S 2.5$. Then $\mathbf{P}$ is bounded from $F M_{0}^{n}$ to $F M_{0}^{n}$. Moreover,

$$
\|\mathbf{P} f\|_{F M^{n}} \leq\|f\|_{F M^{n}} \text { for all } f \in F M_{0}^{n} .
$$

(ii) Let $\mathbf{S}=\mathbf{P J P}$ be as in (2.1). Then $\mathbf{S}$ is bounded from $F M_{0}^{3}$ into itself and so is $\exp (\mathbf{S} t)$. Moreover,

$$
\|\exp (\mathbf{S} t) f\|_{F M^{3}} \leq\|f\|_{F M^{3}} \text { for all } f \in F M_{0}^{3}, t \in \mathbb{R},
$$

where $\exp (\mathbf{S} t)=\sum_{k=0}^{\infty} \frac{1}{k !}(t \mathbf{S})^{k}$.

Proof. (i) Since $\sigma(P)=\dot{I}-\xi \otimes \xi /|\xi|^{2}$, it is an orthogonal projection from $\mathbb{C}^{n}$ into itself. Thus its operator norm is one so that $\||\sigma|\|_{\infty}=1$. From (2.6) it now follows the estimate of (i). The property $\mathbf{P} f \in F M_{0}^{n}$ for $f \in F M_{0}^{n}$ is clear.

(ii) Since $\sigma(\mathbf{S})$ is skew symmetric ([1], [3]), $\exp (\sigma(\mathbf{S}) t)$ is an unitary matrix so $|\exp \sigma(\mathbf{S}) t|=1$ as a matrix. Thus (2.6) yields the desired estimate for $\exp (\mathbf{S} t)$. The boundedness of $\mathbf{S}$ follows from boundedness of its symbol with respect to $\xi$. It is clear that its image is in $F M_{0}^{3}$.

\section{Proof of Theorem 1.1}

We work on the integral equation which is formally equivalent to (1.1) of the form

$$
u(t)=\exp (t \Delta) \exp (-\Omega t \mathbf{S}) u_{0}+N(u, u)(t) \text { for } t \geq 0 .
$$

Here $\exp (t \Delta)=e^{t \Delta}$ is the heat semigroup and

$$
N(u, u)(t)=-\int_{0}^{t} \exp ((t-s) \Delta) \exp (-\Omega(t-s) \mathbf{S}) \mathbf{P} \operatorname{div}(u \otimes u)(s) d s .
$$


There are two key estimates.

(i) estimate for linear term : By Lemma 2.5 and 2.9 (ii) we have

$$
\left\|\exp (t \Delta) \exp (-\Omega t \mathbf{S}) u_{0}\right\|_{F M^{3}} \leq\left\|u_{0}\right\|_{F M^{3}} .
$$

(ii) estimate for nonlinear term : Since we have by Lemma 2.5 (iii)

$$
\nabla \cdot \exp (-\Omega t \mathbf{S}) \mathbf{P} \exp (t \Delta) F \in F M_{0} \text { for any } F=\left(F_{i j}\right)_{1 \leq i, j \leq 3} \in F M^{3 \times 3},
$$

applying Lemma 2.9 together with Lemma 2.5 (iii) yields

$$
\begin{aligned}
& \|\exp (t \Delta) \exp (-\Omega t \mathbf{S}) \mathbf{P} \operatorname{div} F\|_{F M^{3}} \quad\left(\operatorname{div} F=\sum_{i=1}^{3} \partial_{x_{i}} F_{i j}\right) \\
\leq & \|\exp (\Omega t \mathbf{S}) \mathbf{P} \nabla \cdot \exp (t \Delta) F\|_{F M^{3}} \\
\leq & 1 \cdot 1 \cdot\|\nabla \cdot \exp (t \Delta) F\|_{F M^{3}} \\
\leq & (2 t e)^{-1 / 2} \sum_{i=1}^{3}\left\|F_{i}\right\|_{F M^{3}} \quad, \quad F_{i}=\left(F_{i 1}, F_{i 2}, F_{i 3}\right)
\end{aligned}
$$

for all $F \in F M^{3 \times 3}$.

This estimate enables us to construct the solution of the integral equation (3.1) by a successive approximation $\left\{u_{j}\right\}_{j=1,2, \cdots}$ given by

$$
\begin{aligned}
& u_{1}(t)=\exp (t \Delta) \exp (-\Omega t \mathbf{S}) u_{0} \\
& u_{j+1}(t)=u_{1}(t)+N\left(u_{j}, u_{j}\right)(t) \quad j=1,2, \cdots .
\end{aligned}
$$

We now define

$$
K_{j}(T)=\sup _{0 \leq s \leq T}\left\|u_{j}(s)\right\|_{F M}, L_{j}(T)=\sup _{0 \leq s \leq T}\left\|u_{j}(s)-u_{j-1}(s)\right\|_{F M}
$$

for $T>0$. Applying (i), (ii), we have

$$
K_{j+1} \leq\left\|u_{0}\right\|_{F M^{3}}+(2 e)^{-1 / 2} \int_{0}^{t}(t-s)^{-1 / 2} \sum_{i=1}^{3}\left\|F_{i}\right\|_{F M^{3}} d s \quad(j \geq 1)
$$

with $F_{i}=u_{i} u_{j}$. By Lemma 2.4 and the equivalence of norms we observe

$$
\begin{aligned}
& \|v u\|_{F M^{3}} \leq \sqrt{3}\|v u\|_{F M^{3}}^{\prime} \\
& \leq \sqrt{3}\|v\|_{F M}\|u\|_{F M^{3}}^{\prime} \leq \sqrt{3}\|v\|_{F M}\|u\|_{F M^{3}}
\end{aligned}
$$

for $u \in F M^{3}, v \in F M$ so

$$
\sum_{i=1}^{3}\left\|F_{i}\right\|_{F M^{3}} \leq \sqrt{3} \sum_{i=1}^{3}\left\|u_{i}\right\|_{F M}\|u\|_{F M^{3}} \leq 3\|u\|_{F M^{3}}^{\prime}\|u\|_{F M^{3}} \leq 3\|u\|_{F M^{3}}^{2} .
$$

We thus obtain

$$
K_{j+1} \leq\left\|u_{0}\right\|_{F M^{3}}+3(2 / e)^{-1 / 2} T^{1 / 2} K_{j}^{2} \quad(j \geq 1) .
$$


The remaining argument is now standard e.g. [3]. From this estimate one easily sees the uniform boundedness $\sup _{j \geq 1} K_{j}(T) \leq 2\left\|u_{0}\right\|_{F M}$ if $T<1 /\{4$. $\left.3(2 / e)^{-1 / 2}\left\|u_{0}\right\|_{F M^{3}}\right\}^{2}$. Similar calculation for $u_{j+1}-u_{j}$ and the uniform boundedness give us

$$
L_{j+1} \leq 2 \cdot 3(2 / e)^{-1 / 2}\left(2\left\|u_{0}\right\|_{F M^{3}}\right) T^{1 / 2} L_{j} \text { for } j \geq 1,
$$

that implies $L_{j+1} / L_{j}<1 / 2$ if $T \leq 1 /\left[2\left\{2 \cdot 3(2 / e)^{-1 / 2}\left(2\left\|u_{0}\right\|_{F M^{3}}\right)\right\}^{2}\right]$. Therefore, we see there exists a unique limit $u$ such that $u_{j} \rightarrow u$ in $C\left(\left[0, T_{0}\right] ; F M_{0}\right)$ as $j \rightarrow \infty$. It is easy to see that the limit $u$ solves the equation. Uniqueness comes from similar calculation for the subtraction $w=u-v$. Theorem 1.1 has been proved

Remark 3.1. From the above proof the existence time of the solution $u$, denoted as $T_{0}$ in Theorem 1.1, is estimated from below as follows:

$$
T_{0} \geq 1 /\left[2\left\{2 \cdot 3(2 / e)^{-1 / 2}\left(2\left\|u_{0}\right\|_{F M^{3}}\right)\right\}^{2}\right]=e /\left(576\left\|u_{0}\right\|_{F M^{3}}^{2} .\right.
$$

Acknowledgement : The work of the first author is partly supported by the Grant-in-Aid for Scientific Research, No. 14204011, 17654037, the Japan Society of the Promotion of Science (JSPS). The work of the second author was done when he was a post-doctoral fellow at Keio University sponsored by COE 'Integrative Mathematical Sciences: Progress in Mathematics Motivated by Natural and Social Phenomena' (JSPS). Its hospitality is gratefully acknowledged. The work of the third author is partly supported by the AFOSR Contract FG9620-02-1-0026 and the US CRDF Contract RU-M1-2596-ST04. The work of the last author is partly supported by the Grant-in-Aid for Scientific Research, No. 17540201, JSPS.

\section{A Appendix}

We shall prove that the space $F M_{0}$ is strictly included in the Besov space $\dot{B}_{\infty, 1}^{0}$.

Theorem A. The space $F M_{0}$ is continuously embedded in $\dot{B}_{\infty, 1}^{0}$. Moreover, the inclusion $F M_{0} \hookrightarrow \dot{B}_{\infty, 1}^{0}$ is strict.

Proof. For $f \in F M_{0}$ we get

$$
\begin{aligned}
\|f\|_{\dot{B}_{\infty, 1}^{0}} & =\sum_{j=-\infty}^{\infty}\left\|\phi_{j} * f\right\|_{\infty}=\sum_{j=-\infty}^{\infty}\left\|F^{-1}\left(\hat{\phi}_{j} \hat{f}\right)\right\|_{\infty} \\
& \leq C \sum_{j=-\infty}^{\infty}\left\|\hat{\phi}_{j} \hat{f}\right\|_{M} \leq C \sum_{j=-\infty}^{\infty}\left\|\hat{\phi_{j}} \hat{f}\right\|_{M\left(E_{j}\right)} \\
& \leq 3 C\|\hat{f}\|_{M}=3 C\|f\|_{F M} .
\end{aligned}
$$


Here, $E_{j}=\left\{2^{j-1} \leq|\xi| \leq 2^{j+1}\right\}$. Hence the embedding is continuous. The inclusion is strict. We shall prove it for $n=1$. Consider $f=h * e^{i x} i \operatorname{sgn} x \in$ $L^{\infty}(\mathbb{R})$, where $h$ is a smooth bounded function whose Fourier transform $\hat{h}$ is supported in $\{\xi \in \mathbb{R} ;|\xi-1| \leq 1 / 2\}$. Here $\operatorname{sgn}(s)$ is the signature function with value 1 if $s>0$ and -1 otherwise. The Fourier transform $\hat{f}$ equals a constant multiple of $\hat{h}$ p.v. $1 /(\xi-1)$, which is not in $M$; here p.v. denotes the Cauchy principal value. So $f$ does not belong to $F M$. However, since the support of $\hat{f}$ is in $\{|\xi-1| \leq 1 / 2\}$, away from the origin and infinity, the sum $\sum \phi_{j} * f$ in the Besov norm is finite. Since $f \in L^{\infty}(\mathbb{R})$, this implies $f \in B_{\infty, 1}^{0}$. For general $n$ it suffices to consider $F=f\left(x_{1}\right) \cdots f\left(x_{n}\right)$ to observe that $F \notin F M$ but $F \in \dot{B}_{\infty, 1}^{0}$.

\section{References}

[1] A. Babin, A. Mahalov and B. Nicolaenko (1997), Global regularity and integrability of 3D Euler and Navier-Stokes equations for uniformly rotating fluids, Asymptotic Analysis, 15, p. 103-150.

[2] A. Babin, A. Mahalov and B. Nicolaenko (1999), Global regularity of the 3D Rotating Navier-Stokes Equations for resonant domains, Indiana University Mathematics Journal, 48, No. 3, p. 1133-1176.

[3] Y. Giga, K. Inui, A. Mahalov and S. Matsui, Navier-Stokes Equations in a rotating frame in $\mathbb{R}^{3}$ with initial data nondecreasing at infinity, Hokkaido Math. J., to appear.

[4] Y. Giga, A. Mahalov and B. Nicolaenko, The Cauchy problem for the Navier-Stokes equations with spatially almost periodic initial data, to appear in Annals of Mathematical Studies, Princeton University.

[5] M. Hieber and O. Sawada (2005), The Navier-Stokes equations in $\mathbb{R}^{n}$ with linearly growing initial data, Arch. Rational Mech. Anal., 175, no. 2, p. 269-285.

[6] A. Mahalov and B. Nicolaenko (2003), Global regularity of the 3D NavierStokes equations with weakly aligned large initial vorticity, Russian Math. Surveys, 58, No. 2 (350), p. 287-318.

[7] A. Mahalov, B, Nicolaenko, C. Bardos and F. Golse (2004), Regularity of Euler Equations for a class of three-dimensional initial data, Special Volume on Trends in Partial Differential Equations of Mathematical Physics, Nonlinear Analysis Series, Birkhauser-Verlag, 61, p. 161-185.

[8] A.S. Monin and A.M. Yaglom (1971), Statistical fluid mechanics : mechanics of turbulence, volume 1 and 2, MIT Press, Cambridge, Mass.

[9] O. Sawada, The Navier-Stokes flow with linearly growing initial velocity in the whole space, Bol. Soc. Paran. Mat., to appear.

[10] L. Simon (1983), Lecture on geometric measure theory, Proc. of the Center for Math. Anal., Australian National University. 
[11] M.J. Vishik and A.V. Fursikov (1988), Mathematical problems of statistical hydromechanics, Kluwer Academic Publishers, Dordrecht, Netherlands, Boston. 